\title{
Correction to: LIN28B increases neural crest cell migration and leads to transformation of trunk sympathoadrenal precursors
}

\author{
Diana Corallo $\mathbb{D}^{1} \cdot$ Michael Donadon ${ }^{1} \cdot$ Marcella Pantile $^{1} \cdot$ Viktoryia Sidarovich $^{2} \cdot$ Simona Cocchi $^{2} \cdot$ Michela Ori $^{3}$. \\ Miriam De Sarlo ${ }^{3}$. Simona Candiani ${ }^{4}$. Chiara Frasson ${ }^{5} \cdot$ Martin Distel $^{6} \cdot$ Alessandro Quattrone $^{2} \cdot$ Carlo Zanon $^{5}$. \\ Giuseppe Basso ${ }^{7} \cdot$ Gian Paolo Tonini ${ }^{1} \cdot$ Sanja Aveic $\mathbb{D}^{1,8}$
}

Published online: 18 November 2019

(c) The Author(s), under exclusive licence to ADMC Associazione Differenziamento e Morte Cellulare 2019

\section{Correction to: Cell Death \& Differentiation}

https://doi.org/10.1038/s41418-019-0425-3
Since online publication of this article, the authors noticed that there was an error in Supplementary Fig. 6. The figure has now been corrected. The authors apologise for any inconvenience caused.

The original article can be found online at https://doi.org/10.1038/ s41418-019-0425-3.

Diana Corallo

d.corallo@irpcds.org

$\triangle$ Sanja Aveic

s.aveic@irpcds.org

1 Neuroblastoma Laboratory, Fondazione Istituto di Ricerca

Pediatrica Città della Speranza, Padova, Italy

2 Department of Cellular, Computational and Integrative Biology (CIBIO), University of Trento, Trento, Italy

3 Unit of Cell and Developmental Biology, Department of Biology, University of Pisa, Pisa, Italy

4 Department of Earth, Environmental and Life Sciences (DISTAV), University of Genoa, Genova, Italy

5 Fondazione Istituto di Ricerca Pediatrica Città della Speranza, Padova, Italy

6 Innovative Cancer Models, Children's Cancer Research Institute (CCRI), Wien, Austria

7 Department of Women and Child Health, Haematology-Oncology Clinic, University of Padua, Padova, Italy

8 Department of Dental Materials and Biomaterials Research, RWTH Aachen University Hospital, Aachen, Germany 